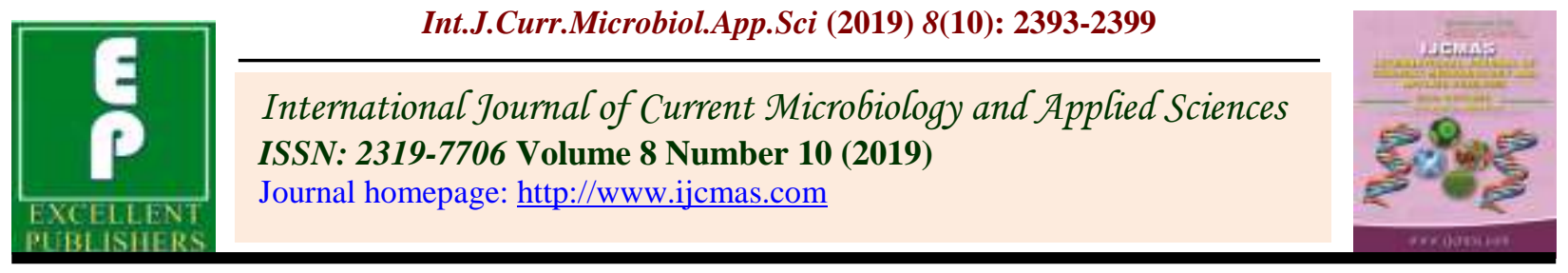

Original Research Article

https://doi.org/10.20546/ijcmas.2019.810.277

\title{
Endoscopic Evaluation of Lower Urinary Tract Affections in Male Dogs
}

\author{
R.N. Nathani*, S.V. Upadhye, P.T. Jadhao, S.B. Akhare, P. Taksande, \\ G.S. Khante and A.M. Kshirsagar \\ Department of Veterinary Surgery \& Radiology, Nagpur Veterinary College, \\ Seminary Hills, Nagpur, India \\ *Corresponding author
}

\author{
A B S T R A C T
}

\begin{abstract}
Keywords
Urinary tract affections, Male dogs

Article Info

Accepted: 17 September 2019 Available Online: 10 October 2019

A study on Endoscopic evaluation of lower urinary tract affections in male dogs was carried out at Teaching Veterinary Clinical Complex of Nagpur Veterinary College, Nagpur. Total 21 dogs with various lower urinary tract affections were evaluated. Dogs presented with clinical signs such as dysuria with dribbling urine, distended bladder, haematuria, oliguria, stranguria, anuria, foul smell in urine, inappetance, vomiting etc. were subjected to endoscopic examination of the lower urinary tract that aided visualisation of various conditions such as urolithiasis, cystitis, urethral stricture and neoplasm. Endoscopic guided basket retrieval of small uroliths was also possible in few cases thereby avoiding need of surgical intervention. Endoscopic guided biopsies were also taken from suspected lesions of bladder that helped in providing confirmatory diagnosis.
\end{abstract}

\section{Introduction}

Different affections that change the normalcy of the lower urinary tract include urolithiasis, upper urinary tract infections, cystitis, urethritis, urinary tract neoplasms, prostate enlargement/ prostatic abscess/prostatic cyst, urethral strictures, extensive or traumatic urethral injuries and ectopic ureters. All these diseases require early diagnosis and prompt management to decrease the morbidity and mortality rates in companion animals. The need of the modern era is to increase the efficacy of diagnostic and therapeutic measures to contribute significantly to the emerging veterinary world.
Urethrocystoscopy is the visualization of the urethral opening, urethra, bladder and ureteral openings. Endoscopic examination has become popular as an innovative technique and frequently used for diagnosing diseases of lower urinary tract. Evaluation of the inside of an organ as well as precise collection of material for further examination can be easily carried out via this advanced technique (Cannizzo et al., 2001).

Endoscopic examination of the urethra and urinary bladder is frequently indicated in dogs with urinary incontinence, hematuria, pollakiuria, stranguria, urinary tract infections, suspected cases of urethral stenosis, chronic 
inflammation, cystoliths, ectopic ureters, bladder diverticula, neoplastic tumours, polyps or cysts of the urinary tract (Grzegoryet al., 2013).

Modernization has led to the development of advanced techniques such as basket retrieval of urinary calculi and laser lithotripsy guided by cystoscopy which are used now-a- days that eliminate any surgical intervention urethrotomy or cystotomy to remove the calculi. Bladder neoplasms were traditionally managed by complete or partial cystectomy, however urethrocystoscopic guided biopsy samples sent for histopathology examination give confirmatory diagnosis and cystoscopic guided coagulation of growths in lower urinary tract is gaining popularity in veterinary science. Also, urethral strictures are now-adays being managed by application of nitinol stents, also guided by trans-urethral cystoscopy (Maggiore et al., 2013).

Considering the growing importance of modern and advanced techniques in the veterinary world, the investigation was carried out to evaluate the efficacy of urethrocystoscopy to diagnose lower urinary tract affections in male dogs.

\section{Materials and Methods}

The study was conducted on the Endoscopic evaluation of lower urinary tract affections in male dogs. The dogs with complaints related to lower urinary tract affections such as hematuria, dysuria, complete or partial urinary obstruction reported to the Teaching Veterinary clinical Complex, Nagpur Veterinary College, Nagpur during the period December 2018 to July 2019 were included in the study. The cases of urinary tract affections as evident from the history were subjected to thorough clinical examination and diagnosis was confirmed through urethrocystoscopy. Thus, a total of 12 cases suffering from various affections of lower urinary tract were included for this study. The cases included cystitis, urolithiasis, urethral stricture and bladder neoplasm.

Urethrocystoscopy is the procedure wherein a flexible or rigid endoscope is passed through the external urethral orifice for visualization of urethra and urinary bladder that aids in diagnosing affections of the lower urinary tract and can also be used as a guiding tool for various therapeutic protocols and collecting biopsy samples for further diagnosis. During the present investigation, urethrocystoscopy was undertaken for diagnosing various affections and also attempts were made to make use of this modality for further confirmative diagnosis and management of some conditions.

\section{Instrumentation and disinfection}

Following instruments/accessories were used to perform urethrocystoscopy in dogs during the study.

1. Monitor

2. Video camera

3. Camera head

4. Fibre-optic light cable

5. Light source

6. $2.7 \mathrm{~mm}$ flexible fibreoptic endoscope, $100 \mathrm{~cm}$ in length

7. Flexible cleaning brush

8. Biopsy forceps-oval, flexible, double action jaws, diameter $1 \mathrm{~mm}$ and length $110 \mathrm{~cm}$

9. Grasping forceps- flexible, double action jaws, diameter $1 \mathrm{~mm}$ and length $110 \mathrm{~cm}$

10. Basket catheter- $115 \mathrm{~cm}$ in length

The disinfection of the endoscope and other instruments was achieved by washing of urethrocystoscope by using distilled water and dipping it in cidex solution (Activated 
glutaraldehyde) for 12 hours prior to use followed by rinsing it with the same and then washing and flushing it again with distilled water.

\section{Urethrocystoscopy procedure}

A flexible urethrocystoscope with $2.7 \mathrm{~mm}$ diameter was used for examination of the lower urinary tract. The procedure was undertaken in male dogs showing clinical symptoms such as hematuria, stranguria, pollakiuria, dribbling urine or complete or partial urinary obstruction.

The dogs were sedated using inj. Xylazine $\mathrm{HCl} @ 1.1 \mathrm{mg} / \mathrm{Kg}$ body weight and positioned in ventro-dorsal recumbency. The whole ventral abdomen, perineum and ischial area were prepared for aseptic endoscopy. Sterile flexible endoscope was passed aseptically into the urethra via the tip of glans penis and advanced gradually while examining the urethral mucosa carefully. Lignocaine hydrochloride gel was applied on tip of penis and endoscope for lubrication and for gentle advancement of the scope in the urinary tract. Urethra was examined for presence of any abnormalities such as haemorrhages, calculi, septic membranes, strictures etc. that could be clearly perceived on screen. Attempts were made to retrieve small sized calculi via basket catheter. The basket catheter was advanced through the instrument port of endoscope until it could be seen on monitor. The tip of the basket catheter was passed over the obstructing urethral calculi and then basket was manually opened to grab the calculus and then closed. The whole assembly (urethrocystoscope along with the basket catheter) was retrieved. Other small sized calculi were retrieved in the similar manner. For thorough visualization of the bladder, urine was first evacuated by catheterization. Entire urinary passage was flushed with normal saline and bladder was distended with 50-100ml of normal saline.

Endoscope was then advanced gradually until it reached the neck of the bladder. The trigone was carefully examined. Complete examination of the bladder was performed by moving the tip of the scope in different directions and advancing and retracting the scope. Suspected tissue specimen from the bladder wall was collected by flexible biopsy forceps, advanced through the instrument port of the endoscope. During the entire procedure, scope was intermittently flushed by normal saline to give a clear view and to avoid hindrance in visualization caused due to bubbles.

\section{Results and Discussion}

During the present investigation, urethrocystoscopy was performed in dogs with various clinical signs related to lower urinary tract affections. Grzegory et al. (2013) also indicated endoscopy of urinary tract in patients with urinary incontinence, hematuria, pollakiuria, stranguria, urinary tract infections, suspected cases of urethral stenosis, chronic inflammation, cystoliths, ectopic ureters, bladder diverticula, neoplastic tumours, polyps or cysts etc. Although endoscopy has established itself as an important diagnostic and therapeutic modality, it has a potential to add the infection to the organ of its use. Therefore the endoscope needs to be sterile to avoid chances of iatrogenic infection. Immersing urethrocystoscope in activated gluteraldyde solution for 5 hours prior to use helped in proper disinfection of the endoscope and instruments as evident from no postendoscopy infection related complications. Grzegory et al. (2013) documented that careless disinfection of endoscopic equipment might induce ascending urinary tract infection as a possible complication. The dogs subjected to endoscopy were sedated using Xylazine injection and local anaesthetic jelly was 
applied over the scope and glans and the dogs were positioned in ventro-dorsal recumbency. Sen et al. (2018) advocated the use of premedication with fentanyl and induction using propofol for cystoscopy in dogs. General anaesthesia is usually recommended for cystoscopic examination (Sen et al., 2018). However, during the present investigation endoscopy of urinary tract could be easily performed under sedation and surface analgesia in dogs wherein no other surgical intervention was required. In cases of urolithiasis, wherein urethrotomy and cystotomy were to be performed, the endoscopic evaluation and surgical interevention was carried out under dissociative anaesthesia. During the present study, a $2.7 \mathrm{~mm}$ flexible endoscope was passed after lubricating the tips of glans and endoscope with lignocaine gel through the external urethral orifice that aided in gentle passing with respect to the anatomical positioning and curve of urethra at perineum.

Out of total 25 dogs that required urethrocystoscopy, the endoscope could be passed only in $21 \mathrm{dogs}$, due to larger diameter of the groove of os penis accommodating the urethra. In cases of tiny dogs like Pugs, Shihtzu and Lhasa, the scope could not be advanced post os-penis, due to this difficulty, it is thought that a smaller diameter endoscope is necessary in smaller breeds of dogs. Lhermette et al. (2015) also mentioned that the size of endoscope that could be passed depended upon the os-penis and flexible endoscope was recommended in male dogs because of the length of the urethra and they further recommended use of sterile watersoluble gel to lubricate the insertion tube and reduce trauma to the mucosa of the urinary tract. Lignocaine gel, used during the present investigation had an added advantage to provide local anaesthesia and easy passing of scope without causing any discomfort. Normal saline was intermittently flushed to clear the passage to visualise urethra normally and avoid any hindrance due to hematuria, blood clots etc. as also recommended by Lhermette et al. (2015). The abnormalities such as urethritis and blood clots in urethra could be seen. Urethral calculi were evident in various cases caudal to os-penis, some of the urethral calculi were snugly fitted obstructing further advancement of the scope. In two cases, these calculi could be retrieved by basket catheter inserted through the instrument port of the endoscope, thereby eliminating the need of surgical intervention since in these cases, the calculi were present only in urethra. Defarges et al. (2013) also opined that calculi too large in size to be retrieved by voiding urohydropropulsion but small enough to be withdrawn from bladder and urethra through gentle traction could be retrieved via stone basket. However, in many cases, although the urethral calculi were located and seen through the endoscope, they could not be retrieved using the basket catheter owing to their large sizes or inability to pass the tip of basket catheter posterior to the calculus since they were snugly lodged in the urethral lumen. Lulich et al. (2016) recommended that urocystoliths small enough to pass through the urethra should be removed by medical dissolution or minimally invasive techniques like voiding urohydropropulsion and basket retrieval to avoid conventional surgeries and added that these procedures had advantages such as less period of hospitalization, shorter anaesthetic time and faster recovery. They also mentioned that the risk of suture induced urolith recurrence was eliminated by avoiding cystotomy. The authors also recommended that smooth uroliths diagnosed in patients with no clinical signs should be removed by minimally invasive techniques as a precautionary measure that might lead to threatening urethral obstruction later in life, if not removed. After removal of urethrolith via basket retrieval, scope was further advanced and urethra was simultaneously carefully 
observed to localise any lesion. Urethritis was a common finding in most of the cases depicted by mucosal haemorrhages. The haemorrhages were seen as petechial, ecchymotic in nature or blood clot based on the extent of the damage caused. In one case, a Doberman was presented with dysuria, wherein prior ultrasound examination revealed prostate enlargement along with prostatic abscess. Endoscopic examination of lower urinary tract in this case revealed urethral stricture at the prostatic urethra and it was difficult to further advance the endoscope. Frequent attempts were made to pass a catheter of larger diameter lubricated with lignocaine gel to dilate urethra in this case. In this case the prostate that was enlarged and had cystic lesions, was aspirated percutaneously under ultrasound guidance. The aspiration of prostate helped in reducing the size of prostate and thereby the pressure on the prostatic urethra.

The passage of serially increasing diameter catheters during the present investigation was sufficient to dilate the urethral lumen. This case was treated with a course of antibiotic after assessing the AST results.

A case of traumatic penile urethral stricture in a dog was managed with placement of a self expanding, covered nitinol stent under cystoscopic guidance by Maggiore et al. (2013). However, during the present investigation, evacuation of prostatic abscess, course of antibiotic and passing catheters of increasing diameter yielded good results and the dogs showed no difficulties in passing the urine thereafter over the period of observation period.

Concentrated urine or urine mixed with blood hindered visualisation of bladder. Hence, bladder was evacuated by catheterisation and it was filled with normal saline that facilitated better examination of bladder and it's affections. Grzegory et al. (2013) also stated that that evacuation of urine from bladder and insufflating it with gas or filling it with saline offered a clearer view of bladder mucosa and aided in better visualisation.

Various bladder lesions such as cystitis, mechanical damage to mucosa due to irritation by uroliths or gravels were evident as inflammation sites with haemorrhages and engorgement of bladder vessels. Uroliths were also seen in the bladder; in one case urolith was retrieved by basket catheter. Lhermette et al. (2015) and Sen et al. (2018) also stated urethrocystoscopy to be the gold standard for diagnosis of urolithiasis and small calculi could be removed using basket forceps transurethrally in male dogs.

Bladder mucosa biopsies were taken using biopsy forceps in two cases from severely inflamed lesions, however the histopathology reports revealed them to be sections of collagenous tissue with no epithelial tissue, thus offering no conclusive pathology.

It is opined that the inflammation at this vesico-ureteric orifice might have been caused due to descend and lodgement of a comparatively larger calculus at this point, the calculus then slipped down into the bladder. Sen et al. (2018) concluded that cystoscopy was more functional than other diagnostic tools with respect to the biopsy samples that could be obtained for histopathological examination, thereby making it more precise and adding influence on further treatment and prognosis of the individual case.

In a non-descript dog that had intermittent hematuria for past 5 months, the urethrocystoscopic examination revealed a cauliflower-like growth with a narrow base and invasion of bladder mucosa. 


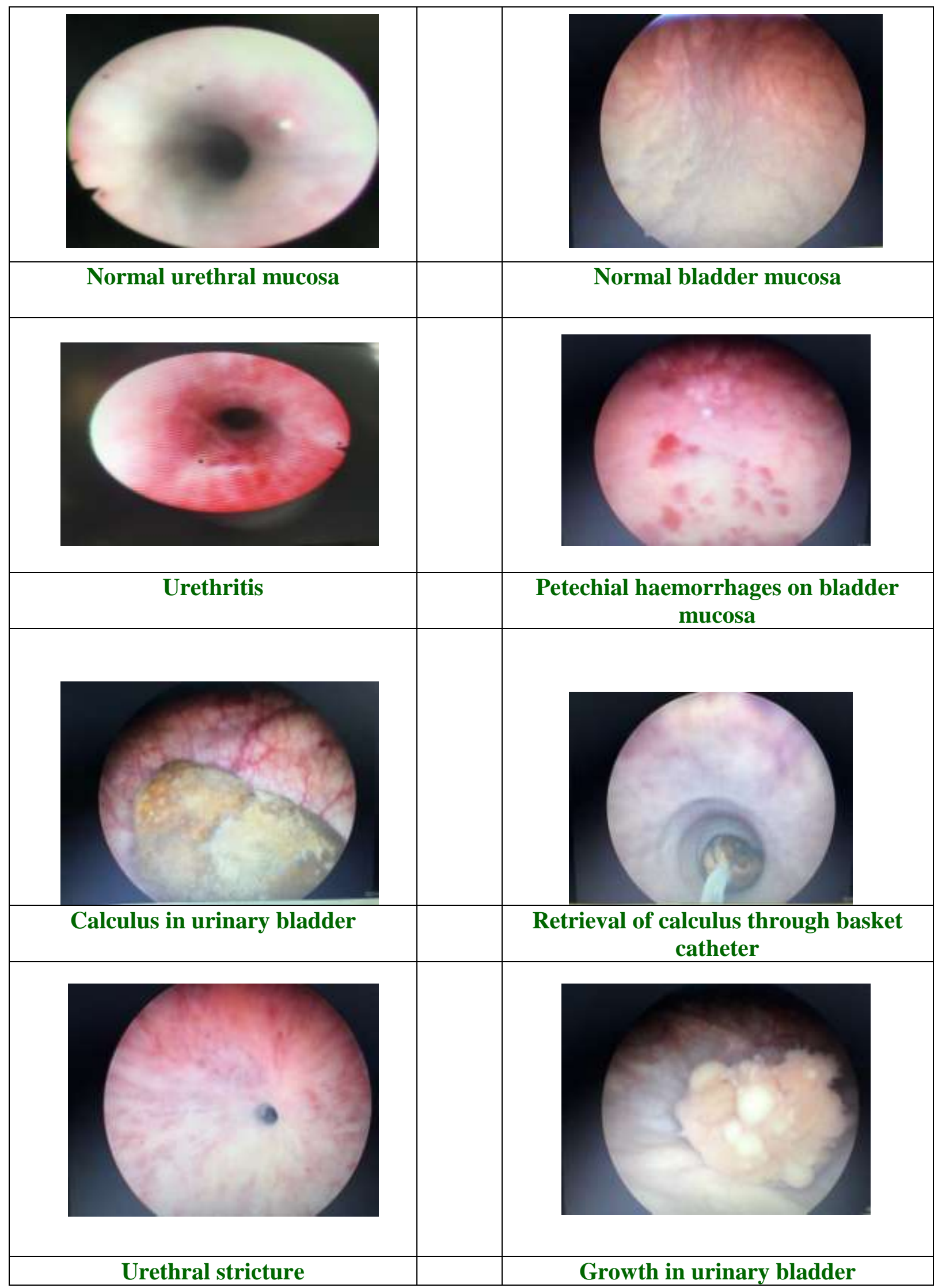


This bladder tumour was diagnosed by ultrasound examination, however, the urethrocystoscopy helped in providing a clear outline of the tumour mass and also revealed the possibility of easy removal by undertaking partial cystectomy. However, considering the clinical condition of the dog, it was decided to perform the surgery after stabilization of the patient. However, the owner did not bring the dog back to the TVCC and therefore, could not be treated as planned.

During the present investigation, three different diagnostic modalities viz. radiography, ultrasound and urethrocystoscopy were utilized. Radiography was useful in diagnosing the conditions like urinary calculi and enlarged prostate. However, in three cases the radiography failed to localize the radiolucent calculi that were then diagnosed by ultrasound examination. The ultrasound examination was very useful diagnostic tool that could diagnose urinary calculi in all cases including urethral calculi, details of prostatic enlargement with abscesses, bladder tumour, cystitis, urinary tract infection as observed due to presence of cellular debris, hydronephrosis and hydroureters. The diagnostic modality urethrocystoscopy was found useful in actual visualization of the calculi, stricture of urethra, bladder tumour, and changes in mucosa of bladder due to cystitis. However, as mentioned earlier, the urethral calculi created hindrance in further passing of the endoscope and thereby restricting further exploration of the urinary tract. Similarly, the extraluminal causes that resulted in urethral obstruction such as enlarged prostrate, prostatic abscess etc could not be diagnosed.

Therefore, it was concluded that although urethrocystoscopy is an excellent modality, it cannot replace radiography and ultrasound for diagnosing various affections of the lower urinary tract.

\section{How to cite this article:}

Nathani, R.N., S.V. Upadhye, P.T. Jadhao, S.B. Akhare, P. Taksande, G.S. Khante and Kshirsagar, A.M. 2019. Endoscopic Evaluation of Lower Urinary Tract Affections in Male Dogs. Int.J.Curr.Microbiol.App.Sci. 8(10): 2393-2399. doi: https://doi.org/10.20546/ijcmas.2019.810.277

\section{References}

Cannizo, K.L., M.A. McLoughlin, D.J. Chew, S.P. Dibartola (2001) Uroendoscopy: Evaluation of the lower urinary tract. Vet Clin North Am Small Anim Pract 31: 789807.

Defarges, A., M. Dunn and A. Berent (2013) New alternatives for minimally invasive management of uroliths: Lower urinary tract uroliths. Compendium Yardley PA: Continuing education for veterinarians.

Grzegory, M., K. Kubiak, M. Jankowski, J. Spuzak, K. Glinska-Suchochka, J. Bakowska, J. Nicpon and A. Halon (2013) Endoscopic examination of the urethra and the urinary bladder in dogs- indications, contraindications and performance technique. Polish Journal of Veterinary Science. 16(4):797-801.

Lhermette, P. (2015) Urethrocystoscopy of the urinary system in dogs and cats. Companion animals. 37 : 445-455.

Lulich, J.P., A.C. Berent, L.G. Adams, J.L. Westropp, J.W. Bartges and C.A. Osborne (2016) ACVIM Small Animal Consensus Recommendation on the Treatment and Prevention of Uroliths in Dogs and Cats. Journal of Veterinary Internal Medicine. 30(5):1564-1574.

Maggiore, A.M.D., M.A. Steffey and J.L. Westropp. (2013) Treatment of traumatic penile urethral stricture in a dog with selfexpanding covered nitinol stent. Journal of the American Veterinary Medical Association. 242(8) : 1117-1121.

Sen, Y., A. Bumin, I. Ekmen and G. Sonmez (2018) Comparison and evaluation of the lower urinary tract diseases radiographic, ultrasonographic, diagnosis and cystoscopic examination findings in dogs. Albanian J. Agric. Sci (special edition - proceedings of ICOALS: 132-137). Vetlearn.com E1-E7 (PMID: 23532727). 